[13] L. Li, J. Q. He, Z. Liu, X. Dong, and L. Carin, "MLFMA analysis of scattering from multiple targets in the presence of a half space," IEEE Trans. Antennas Propagat., vol. 51, pp. 810-819, Apr. 2003.

[14] L. Li, Z. Liu, X. Dong, J. Thompson, and L. Carin, "Scalable multi-level fast multipole method for multiple targets in the vicinity of a half space," IEEE Trans. Geosci. Remote Sensing, vol. 41, pp. 791-802, Apr. 2003.

[15] P. Pathak, W. Burnside, and R. Marhefka, "A uniform GTD analysis of the diffraction of electromagnetic waves by a smooth convex surface," IEEE Trans. Antennas Propagat., vol. AP-28, pp. 631-642, 1980.

[16] G. Zhang, L. Tsang, and Z. Chen, "Collective scattering effects of trees generated by stochastic Lindenmayer systems," Microwave Optical Tech. Letts., vol. 11, pp. 107-111, Feb. 1996.

[17] Y.-C. Lin and K. Sarabandi, "A Monte Carlo coherent scattering model for forest canopies using fractal-generated trees," IEEE Trans. Geosc. Remote Sens., vol. 37, pp. 440-451, Jan. 1999.

\section{Fast Numerical Solution for Scattering From Rough Surfaces With Small Slopes}

\author{
M. Saillard and G. Soriano
}

\begin{abstract}
The rigorous solution of the scattering by two-dimensional rough surfaces can be numerically performed thanks to the sparse matrix flat surface iterative approach (SMFSIA). However, this kind of computation still requires both large CPU time and memory. Consequently, a lot of work has been dedicated at extending the domain of validity of approximate methods beyond that of standard high and low frequency approximations. In this paper it is shown that SMSFIA can be simplified in order to get a fast approximate method, with low memory requirements. This approximation is valid for surfaces with small slopes, and covers the domain of validity of both the Kirchhoff -tangent plane approximation and the first-order small slope approximation [1].
\end{abstract}

Index Terms-Ocean remote sensing, rough surface scattering.

\section{INTRODUCTION}

In most remote sensing applications, scattering from natural surfaces cannot be accurately estimated with the Kirchhoff-tangent plane approximation or with the small perturbation method. For surfaces with very wide spectrum, like sea surface, two-scale models combining both these high and low frequency approximations have been proposed [2], [3]. As a shortcoming, a cutoff frequency separating the two domains has to be chosen, and, since the domain of validity of the two approximations generally do not overlap, the solution depends on this cutoff frequency. In another way, several attempts have been made to improve Kirchhoff approximation or small perturbation method, so that a single approximate method can deal with such multiscale surfaces, leading to the small slope approximation [1], the operator expansion method (OEM) [4], the integral equation method [5], [6], or the extended Kirchhoff [7]. However, to fit both the high and low frequency approximations, the scattering amplitude has to be represented as a double integral over the surface. Computation of such second order terms is a hard task, since it generally involves multiple integration of oscillating functions. In addition, analytical expressions of the scattering amplitudes

Manuscript received April 2, 2002; revised December 6, 2002.

The authors are with the Institut Fresnel UMR-CNRS 6133, Faculte des Sciences et Techniques de St Jérôme, 13397 Marseille Cedex 20, France (e-mail: marc.saillard@fresnel.fr).

Digital Object Identifier 10.1109/TAP.2004.834413 involving surface statistics can only be obtained under the assumption of Gaussian surface height distribution. To overcome these two problems, the second order small slope approximation has been combined with a Monte-Carlo approach in [8], but even in this case, one has to perform a double integration still, one in the spatial domain and one in the spectral domain, for each scattering angle, which makes the computation of the bistatic scattering pattern rather long. It must also be pointed out that such methods do not allow one to estimate the accuracy of the result.

These remarks lead us to reconsider the use of the numerical methods based on Monte-Carlo simulations. The sparse matrix flat surface iterative approach (SMFSIA) [9], [10] is an efficient method for the numerical solution of the rigorous problem of electromagnetic scattering by two-dimensional (2-D) rough surfaces. For perfectly conducting surfaces, the magnetic field integral equation (MFIE) is discretized with a method of moments. The matrix of the resulting linear system is split into a strong matrix, representing close interactions, a flat surface matrix, approximating far interactions, and the remaining weak matrix. The flat surface matrix has been introduced to take benefit from its block-Toeplitz structure. As a consequence, it can be easily stored in central memory, and matrix-vector products are computed very fast (see Section II).

It is important to point out that the operator described by the flat surface matrix coincides with that obtained when the approximation suggested by Meecham and Lysanov [1] is applied to the MFIE. This approximation has already been implemented for the scalar solution of Helmholtz equation with Dirichlet boundary condition. In this case, it coincides with the operator expansion method at lowest order and it is known to be accurate for moderate slopes and heights. When the 3-D electromagnetic problem is addressed, though similarities remain, the two approaches are no longer identical. Indeed, in [4], the author expresses the scattered field as a single layer potential (convolution product of Green's function with some surface density), while Stratton-Chu formula are used here to derive the scattered field. Therefore, the method described in this paper and OEM at lowest order apply the same approximation but to different integral equations. The way these equations are solved also differ: an approximate analytical expression is derived in [4] and a (fast) numerical solution is proposed here.

Therefore, we suggest to drop the weak matrix and its associated iterative level in the SMSFIA. Such a suggestion was first made in [11] in the frame of the 2-D scalar Helmholtz equation with Dirichlet boundary condition, but was not further investigated, since it coincides with OEM in this case. Here, this approximation has been applied to surfaces with Gaussian correlation function and to a multiscale surface with band-limited power-law spectrum, then compared to the SMFSIA, and to the Kirchhoff, small slope, and small perturbation approximations.

\section{FORMULATION}

Let us consider an incident electromagnetic field $\mathbf{E}^{0}, \mathbf{H}^{0}$ in the vacuum, impinging on a rough surface $\Sigma$ with Cartesian equation $z=h(\mathbf{r})=h(x, y)$. We denote by $\sigma$ the rms height and by $s$ the rms slope of the surface. The electromagnetic field is time-harmonic, and an $\exp (-i \omega t)$ time dependence is assumed. The surface is of infinite extent, but is illuminated by a Gaussian beam, so that only a finite area has to be considered. The scattered field is denoted by $\mathbf{E}^{s}, \mathbf{H}^{s}$. For a perfectly conducting surface, the surface current $\mathbf{j}=\hat{\mathbf{n}} \times\left(\mathbf{H}^{0}+\mathbf{H}^{s}\right)$ satisfy the MFIE $(1 / 2+\mathrm{M}) \mathbf{j}=\hat{\mathbf{n}} \times \mathbf{H}^{0}$, where the normal to the surface is directed toward vacuum. $M$ is the integral 
operator introduced by Martin and Ola in [12]. $\mathbf{R}=\mathbf{r}+h(\mathbf{r}) \hat{\mathbf{z}}$ and $\mathbf{R}^{\prime}=\mathbf{r}^{\prime}+h\left(\mathbf{r}^{\prime}\right) \hat{\mathbf{z}}$ being two points on $\Sigma$

$$
\mathrm{M}_{\mathbf{R}}=\hat{\mathbf{n}}_{\mathbf{R}} \times \operatorname{curl}_{\mathbf{R}} \int_{\Sigma} G_{\mathbf{R}, \mathbf{R}^{\prime} \mathbf{j}_{\mathbf{R}^{\prime}} d \Sigma^{\prime}}
$$

where $G_{\mathbf{R}, \mathbf{R}^{\prime}}$ is the free space Green's function with wavenumber $K=$ $\omega / c$. Introducing the scalar function $g(x)=-\exp (i K x) / 4 \pi x$, the horizontal distance $d=\left\|\mathbf{r}-\mathbf{r}^{\prime}\right\|$, the horizontal unit vector $\hat{\mathbf{u}}=\left(\mathbf{r}-\mathbf{r}^{\prime}\right) / d$ and the slope $p=\left(h(\mathbf{r})-h\left(\mathbf{r}^{\prime}\right)\right) / d$, operator M writes

$$
\mathbf{M j}_{\mathbf{R}}=\hat{\mathbf{n}}_{\mathbf{R}} \times \int_{\Sigma} d g^{\prime}\left(d \sqrt{1+p^{2}}\right)(\hat{\mathbf{u}}+p \hat{\mathbf{z}}) \times \mathbf{j}_{\mathbf{R}^{\prime}} d \Sigma^{\prime}
$$

If $K \sigma s$ is small, $g^{\prime}\left(d \sqrt{1+p^{2}}\right)$ can be approximated by $g^{\prime}(d)$, and one finds the flat surface operator $\mathrm{M}_{F S}$, defined in the SMFSIA

$$
\mathrm{M}_{F S} \mathbf{j}_{\mathbf{R}}=\hat{\mathbf{n}}_{\mathbf{R}} \times \int_{\Sigma} d g^{\prime}(d)(\hat{\mathbf{u}}+p \hat{\mathbf{z}}) \times \mathbf{j}_{\mathbf{R}^{\prime}} d \Sigma^{\prime}
$$

as a small slope approximation of M. It is important to notice that the criterion for validity concerns both height and slope, since $K \sigma s$ represents the deviation of the phase from flat to rough surface. This property is shared with the OEM.

Following [9], the MFIE is written as a set of two coupled scalar equations, after projection onto $\hat{\mathbf{x}}$ and $\hat{\mathbf{y}}$. To discretize these equations, a method of moments is applied on a regular 2-D gridding of the $x O y$ plane, with pulse basis functions and delta weighting functions. Diagonal matrix elements are computed separately, using the method described in [13], as applied to (1). These terms corresponds to the strong matrix $\mathcal{A}_{S}$ of SMFSIA with strong interaction radius $r_{d}=0$. All the remaining elements are evaluated according to (2), and are set to the value of the kernel at the center of the element times the area of the element. This way, $d g^{\prime}(d)$ becomes a 2-D Toeplitz matrix. It can be immersed into a 2-D circulant matrix, which is diagonal in the Fourier space. Only these diagonal elements need to be computed and stored. If $N$ is the number of surface unknowns, the strong matrix has $N$ elements, the flat surface matrix $\mathcal{A}_{F S}$ has $4 N$ elements (in Fourier space). For example, the scattering problem associated with a $32 \lambda$ by $32 \lambda$ surface sampled at 8 points per $\lambda$ has $N=65536$ surface sampling points, and thus requires $5 \mathrm{Mb}$ in central memory (if double precision is used).

The approximate linear system $\left(\mathcal{A}_{S}+\mathcal{A}_{F S}\right) \mathbf{X}=\mathbf{B}$ is solved by the biconjugate gradient stabilized of order 2 . Each iteration requires four matrix-vector products. There are 12 fast-Fourier transforms (FFT) per matrix-vector, and $4 N \log 4 N$ operations per FFT.

\section{NUMERICAL RESUlTS AND DISCUSSIONS}

This approximation, which will be referred to as the small slope integral equation (SSIE), is first applied to two perfectly conducting surfaces with Gaussian correlation function (in this section, all surfaces have Gaussian height distribution), at $20^{\circ}$ incidence. In the first example, the rms height is $0.17 \lambda$ and the correlation length is $1 \lambda$. For this surface, $K \sigma s \simeq 0.25$, which has been estimated as the limit of validity of first-order OEM [14] when applied to 1-D surfaces with Dirichlet boundary conditions. In Fig. 1, SSIE is compared to the rigorous computation (SMFSIA) through averaging over 100 surface samples, $24 \lambda \times 24 \lambda$ square. For $V$ incident polarization, SMFSIA and SSIE give extremely close results for both co- and cross-polarization, while some discrepancy occurs for $H$ polarized incident wave, cross-polarization being slightly overestimated. This discrepancy disappears for lower values of $K \sigma s$, leading to perfect agreement
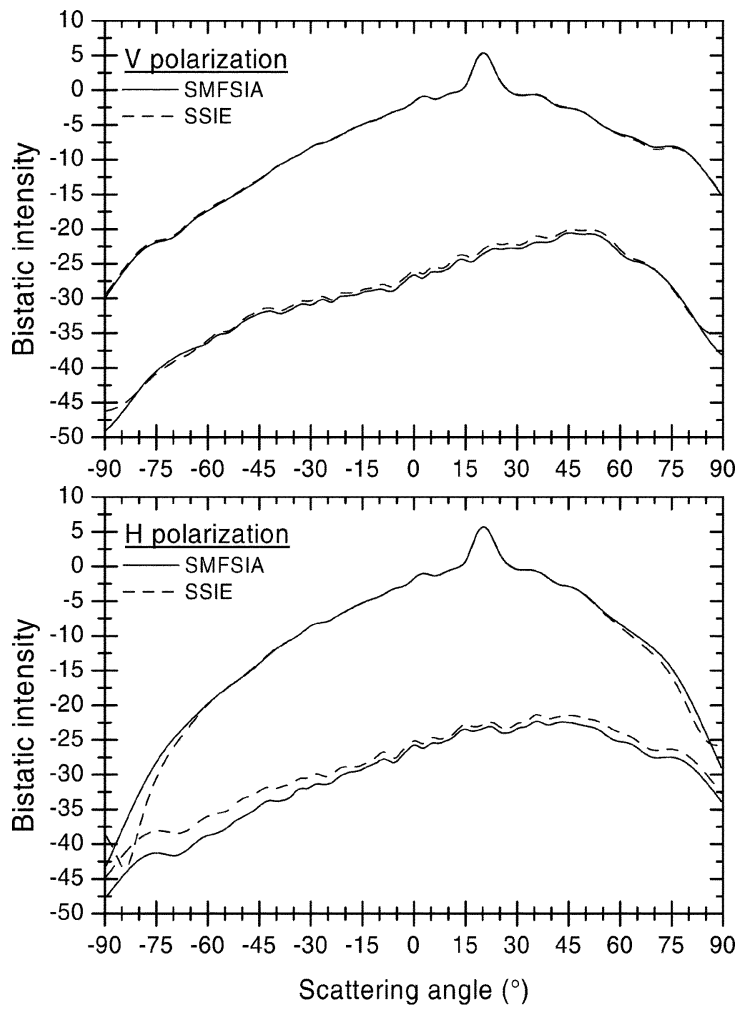

Fig. 1. Scattering diagrams in the plane of incidence for a surface with Gaussian correlation, rms $0.17 \lambda$ and correlation length $1.0 \lambda$ and for an incident angle of $20^{\circ}$. The curves at high energy level represent the co-polarized contribution, while the cross-polarized component is represented by the lower curves of each plot.

between SMFSIA ans SSIE. Contrary to classical first-order approximate methods, solving the approximate linear system permits one to take multiple scattering into account, such that even the low-level cross-polarization in the plane of incidence can be estimated thanks to SSIE. The numerical costs of the two methods can be compared. For this surface with 20736 sampling points (twice more unknowns), the SMFSIA takes $400 \mathrm{Mb}$ of random access memory to store the strong and flat surface matrices and requires $6 \mathrm{~min} \mathrm{CPU}$ time to solve the linear system. The costs of SSIE are $1.6 \mathrm{Mb}$ for the matrices and $30 \mathrm{~s}$ for solution.

The second Gaussian surface considered here has a rms height of $0.5 \lambda$ and a correlation length of $2.0 \lambda$ (Fig. 2). This example is at the limit of the domain of validity of Kirchhoff approximation, while small slope approximation is clearly inaccurate. In principle, since $K \sigma s \simeq$ 1.11 , the phase perturbation should not be neglected. However, SSIE outperforms other approximate methods and remains very close to the rigorous solution for co-polarization, except at grazing scattering angles. On the other hand, cross-polarization is overestimated by several $\mathrm{dB}$. Using this method, one can also get an estimation of the accuracy of the computation. With this aim, the Ewald-Oseen extinction theorem [15] has been tested for each surface sample. It consists in computing from the surface current a scattered field in the lower half-space using the electric permittivity of the vacuum. This field, the extinction field, must be the exact opposite of the incident field. In the last example, even though the statistical result is satisfactory in co-polarization, some computations have exhibited strong deviations from the rigorous result. The worse case observed among the hundred ones used in the average process has lead to $50 \%$ deviation to the extinction theorem. Memory requirements are the same as for the first surface, and SMFSIA solves the linear system in $40 \mathrm{~min}$ while SSIE needs only $90 \mathrm{~s}$. 

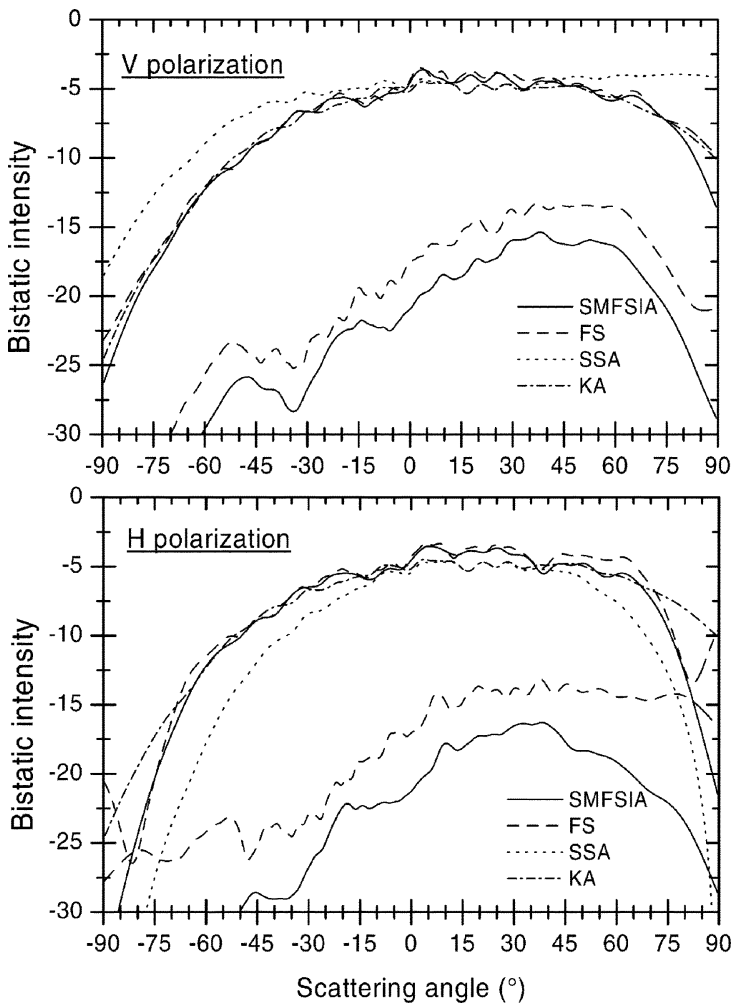

Fig. 2. Same as Fig. 1 for a surface with rms $0.5 \lambda$ and correlation length $2.0 \lambda$.

We have then considered a multiscale surface with a band-limited power-law spectrum: $\Psi_{r}(\boldsymbol{\kappa})=\alpha /\left(2 \pi \kappa^{4}\right)$ for spatial wavenumbers $\kappa \in[K / 30 ; 4 K]$, at $20^{\circ}$ incidence. With $\alpha=5.2510^{-3}$, this spectrum is a good approximation of the high frequency part of the omnidirectional ocean spectrum at $L$ band for a $10 \mathrm{~m} / \mathrm{s}$ wind [16], [17]. This surface has a root-mean-square (rms) height of $\sigma=0.25 \lambda$ and a slope rms of $s=0.112$, leading to $K \sigma s \simeq 0.176$, well suited for this approximation. It has already been shown that electromagnetic scattering from 2-D rough surfaces with large conductivity can be modelized by a single surface integral equation, thanks to a curved-surface impedance boundary condition [18]. As the MFIE for perfect conductors, this equation is weakly singular, we thus can apply both the SMFSIA and the SSIE. It can be seen in Fig. 3 that in co-polarization and for both incident polarizations, SMFSIA, SSIE, and small slope approximation are in good agreement. Here, small perturbation method and Kirchhoff approximation are not reported, but it has been shown in [17] that they are not revelant for such a multiscale surface. SSIE is slightly overestimating the cross-polarized intensity around the specular direction. Here, the computational costs of SSIE are $16 \mathrm{Mb}$ for matrices and 6 min for solution. For the SMFSIA and due to the large number of surface sampling points (82944), a beam decomposition technique with 16 narrow beams is applied [18]. Each one requires $250 \mathrm{Mb}$ and 6 min. To show the versatility of the method, we have also performed computations at $80^{\circ}$ incidence. It is well known that the length of the surface in the direction of the mean incident wavevector has to be dramatically increased at grazing incidence. Here, it has been set to $256 \lambda$. To save computation time we have restricted the width of the surface samples to $16 \lambda$, which is smaller than the upper bound of the surface spectrum, thus leading to artificial geometrical anisotropy. As a consequence, one can notice in Fig. 4 that the two cross-polarized intensities differ in this case. The number of sampling points is 262144 , requiring about $50 \mathrm{~min}$ to solve the linear system on our PC. The extinction theorem remains very accurate, typically $10^{-2}$ to $10^{-3}$.

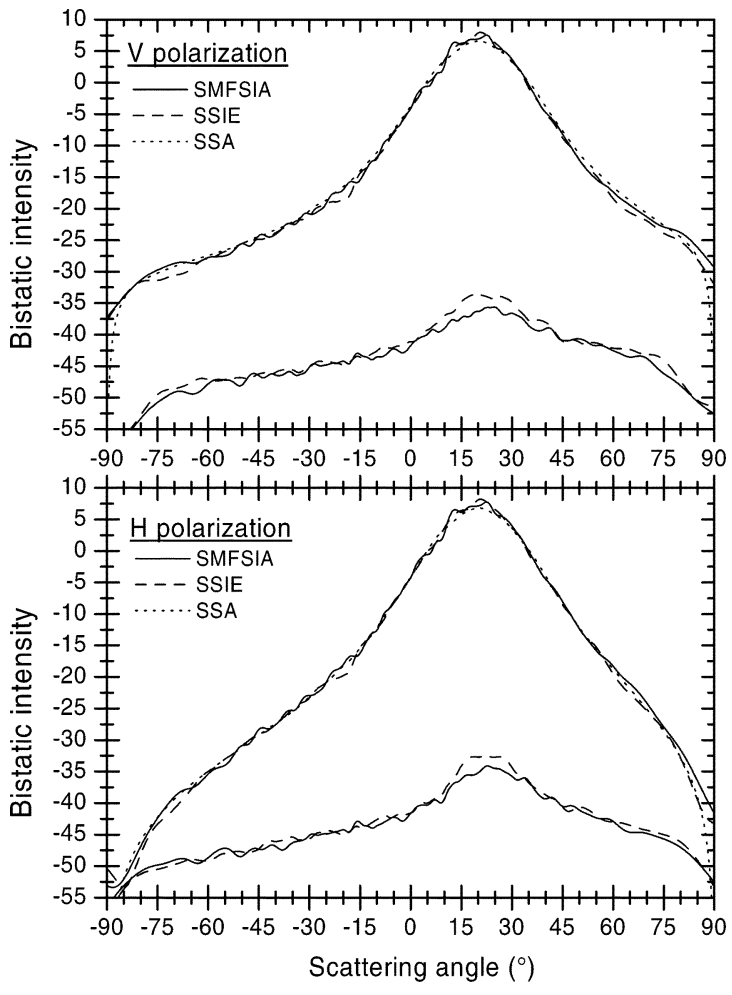

Fig. 3. Same as Fig. 1 for a surface with a $\kappa^{-4}$ band-limited power law spectrum and a permittivity of $73.5+i 61.0$ for an incident angle of $20^{\circ}$.

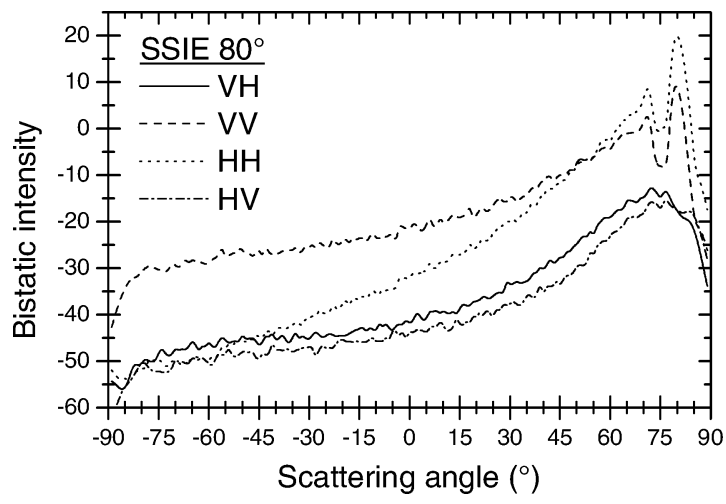

Fig. 4. Same as Fig. 3 for an incident angle of $80^{\circ}$.

\section{CONCLUSION}

To draw a first conclusion, the SSIE approximation appears to have a domain of validity that covers both that of the first-order small slope approximation and of the Kirchhoff-tangent plane approximation. It also provides an estimation of the cross-polarized component in the plane of incidence. This is very similar to the second-order small slope approximation or to the OEM. It has been tested with single scale Gaussian metallic surfaces as well as with multiscale surfaces with power law spectrum. From a theoretical point of view, its extension to dielectric penetrable surfaces is straightforward. As a numerical method based on a Monte-Carlo average process, there is no hypothesis on the surface spectrum or on its height distribution, and the accuracy of the computation can be estimated. In addition, taking benefit from the block Toeplitz structure of the matrix permits one to solve huge linear system at very low computational cost. In our opinion, it is an interesting alternative to statistical approximate methods when second order corrections have to be taken into account. 


\section{REFERENCES}

[1] G. Voronovich, Wave Scattering from Rough Surfaces. Berlin: Springer-Verlag, 1994.

[2] G. R. Valenzuela, "Theories for the interactions of electromagnetic and oceanic waves - A review," Boundary-Layer Meteorology, vol. 13, pp. $61-85,1978$.

[3] F. G. Bass and I. M. fuks, Wave Scattering from Statistically Rough Surfaces. New York: Pergamon, 1979.

[4] D. M. Milder, "An improved formalism for electromagnetic scattering from a perfectly conducting rough surface," Radio Sci., vol. 31, pp. 1369-1371, 1996.

[5] A. K. Fung, Microwave Scattering and Emission Models and their Applications. Boston: Artech House, 1994.

[6] J. L. Alvarez-Perez, "An extension of the IEM/IEMM surface scattering model," Waves in Random Media, vol. 11, pp. 307-329, 2001.

[7] T. Elfouhaily, D. R. Thompson, D. E. Freund, D. Vandemark, and B. Chapron, "A new bistatic model for electromagnetic scattering from perfectly conducting surfaces: Numerical evaluation and comparison with SPM," Waves in Random Media, vol. 11, pp. 33-43, 2001.

[8] S. T. McDaniel, "Acoustic and radar scattering from directional seas," Waves Random Media, vol. 9, pp. 537-549, 1999.

[9] K. P. Pak, L. Tsang, C. H. Chan, and J. Johnson, "Backscattering enhancement of electromagnetic waves from two-dimensional perfectly conducting random rough surfaces based on Monte-Carlo simulations," J. Opt. Soc. Am. A, vol. 12, pp. 2491-2499, 1995.

[10] J. T. Johnson, L. Tsang, R. T. Shin, K. Pak, C. H. Chan, A. Ishimaru, and Y. Kuga, "Backscattering enhancement of electromagnetic waves from two-dimensional perfectly conducting random rough surfaces: A comparison of Monte Carlo simulations with experimental data," IEEE Trans. Antennas Propagat., vol. 44, pp. 748-756, 1996.

[11] J. T. Johnson, "On the canonical grid method for two-dimensional scattering problems," IEEE Trans. Antennas Propagat., vol. 46, pp. 297-302, 1998.

[12] P. A. Martin and P. Ola, "Boundary integral equations for the scattering of electromagnetic waves by a homogeneous dielectric obstacle," in Proc. Roy. Soc. Edinburgh, vol. 123, 1993.

[13] C. Macaskill and B. J. Kachoyan, "Iterative approach for the numerical simulation of scattering from one and two dimensional rough surfaces," Applied Optics, vol. 32, pp. 2839-2847, 1993.

[14] P. J. Kaczkowski and E. I. Thorsos, "Application of the operator expansion method to scattering from one-dimensional moderatly rough Dirichlet random surfaces," J. Acoust. Soc. Ameer., vol. 96, pp. 957-972, 1994.

[15] M. Nieto-Vesperinas and J. C. Dainty, Scattering in Volume and Surfaces: Elsevier Science Publishers, B. V. North-Holland, 1990.

[16] T. Elfouhaily, B. Chapron, and K. Katsaros, "A unified directional spectrum for long and short wind-driven waves," J. Geophys. Res., vol. 102, pp. 15781-15 796, 1997.

[17] G. Soriano, C. A. Guerin, and M. Saillard, "Scattering by two-dimensional rough surfaces: Comparison between the method of moments, Kirchhoff and small-slope approximations," Waves in Random Media, vol. 12 , pp. 63-83, 2002.

[18] G. Soriano and M. Saillard, "Scattering of electromagnetric waves from two-dimensional rough surfaces with impedance approximation," J. Opt. Soc. Amer. A, vol. 18, pp. 124-133, 2001.

\section{Fixing Limits to Free-Access Areas Around Broadcast Antennas}

\author{
Iñigo Cuiñas, Antonio García Pino, Manuel García Sánchez, \\ Marcos Arias, and Alonso A. Alonso
}

Abstract-The growing interest in the relation between electromagnetic fields and human health forces the assumption of national and international regulations on emission levels. In order to reduce the risks, restricted access around transmitting antennas have to be defined according with those regulations. This paper offers a guideline to determine the boundary of the potentially risky volumes around radio broadcasting antennas for AM-MF and FM-VHF services. With the proposed method, a complete reference volume is defined by just five parameters.

Index Terms-Antenna radiation patterns, biological effects of electromagnetic radiation, electromagnetic radiation effects, Pollution.

\section{INTRODUCTION}

Social factors in technologically developed countries have reopened a topic that seemed to be closed some years ago: the influence of electromagnetic radiation on human health. Military standards were adopted to preserve the health of people staying near radar and communications antennas after World War II. However, the expansion of mobile communication networks, and radio-frequency based communication systems in general, has compelled the erection of many antenna towers all around our cities. These antennas are of special concern due to electromagnetic and visual pollution. Latest research results are closely observed by the International Commission on Non-Ionizing Radiation Protection (ICNIRP). This nongovernmental organization, formally recognized by World Health Organization (WHO), evaluates scientific results from all over the world. ICNIRP produces guidelines recommending limits of exposure, which are revised periodically and updated [1]. Some guidelines, developed for electromagnetic fields exposure, covering the nonionizing radiation up to $300 \mathrm{GHz}$ can be found in [2] and [3].

National and international regulations have fixed exposure limits on electromagnetic fields that can be related to safety distances to the transmitting elements [4]. Many governments and administrations adopted the ICNIRPreferencelevels. The establishment of restricted areas around the antenna (by painting nontrespassing lines or by installing fences) is a way to prevent that people will enter a potentially risky place.

In [5], the near field of wireless base-station antennas is studied under the exposure compliance point of view. The case of Medium Frequency (MF) broadcasting is especially interesting because people could be in the surroundings of the antennas, installed at ground level, supporting radiation levels over the WHO-recommended exposure limits. The AM-MF antennas are commonly composed of masts with a height of several tens of meters, radiating as a monopole over ground plane. In this case, the installation of a fence near the antenna is necessary to avoid people touching the mast. In addition, another element (painted line or fence) is needed to limit the presence of people inside the risky area. Furthermore, antennas for FM-VHF broadcast services are usually installed on the same mast.

Manuscript received January 22, 2003; revised October 1, 2003. This work was sponsored by X. de Galicia, under the Environment Technology project PGIDIT02TAM322091PR.

I. Cuiñas, A. G. Pino, M. G. Sánchez, and M. Arias are with the Universidade de Vigo, Dept. Teoría do Sinal e Comunicacións, E36200 Vigo, Spain (e-mail: inhigo@com.uvigo.es).

A. A. Alonso is with the Universidad de Valladolid, Dept. Teoría de la Señal y Comunicaciones e Ingeniería Telemática, Valladolid E47011, Spain.

Digital Object Identifier 10.1109/TAP.2004.834412 